\title{
Review of the British Thoracic Society Winter Meeting 2017, 6-8 December 2017, London, UK
}

\author{
Aran Singanayagam, ${ }^{1}$ Hannah V Woodcock, ${ }^{2}$ Philip L Molyneaux ${ }_{1}^{3,4}$ Gisli Jenkins ${ }^{5}$
}

${ }^{1}$ COPD and Asthma Section, National Heart and Lung Institute, Imperial College London, London, UK

${ }^{2}$ Centre for Inflammation and Tissue Repair, UCL Respiratory, Rayne Institute, University College London, London, UK ${ }^{3} \mathrm{NIHR}$ Respiratory Biomedical Research Unit, Royal Brompton Hospital, London, UK

${ }^{4}$ Fibrosis Research Group, National Heart and Lung Institute, Imperial College London, London, UK

${ }^{5}$ Centre for Respiratory Research, University of Nottingham, Nottingham, UK

\section{Correspondence to}

Professor Gisli Jenkins, Centre for Respiratory Research, University of Nottingham, Nottingham NG5 1PB, UK; gisli.jenkins@nottingham.ac.uk

Received 1 May 2018 Revised 14 May 2018 Accepted 21 May 2018 Published Online First

14 June 2018

Check for updates

To cite: Singanayagam A, Woodcock HV, Molyneaux PL, et al. Thorax

2018:73:872-876.

\section{ABSTRACT}

This article reviews the British Thoracic Society Winter Meeting 2017 and summarises the new developments in scientific and clinical research across the breadth of respiratory medicine. The article discusses a number of symposia and selected abstract presentations from the meeting.

\section{INTRODUCTION}

The British Thoracic Society (BTS) Winter Meeting 2017 attracted 2300 delegates to London. The meeting showcased the latest advances in respiratory science including clinical and translational research. This article summarises selected key sessions.

\section{BTS/BRITISH LUNG FOUNDATION/BRITISH ASSOCIATION OF LUNG RESEARCH EARLY CAREER INVESTIGATORS SYMPOSIUM}

This exciting session, featuring six emerging early career academics, provided a glimpse at the future of UK academic respiratory medicine. Dr Wortley (London, UK) won the BTS award for his research that used in vivo and ex vivo experimental approaches to demonstrate that bacteria can activate sensory nerves, specifically via TLR-2. ${ }^{1}$ These effects could explain increased coughing episodes that occur during acute bacterial infection. Runner up for this award was Dr Walstead (London, UK and Copenhagen, Denmark) who presented data from six subjects with exercise-induced laryngeal obstruction showing that respiratory work and neural drive increases in close association with paradoxical laryngeal closure in this condition. ${ }^{2}$

Dr Wood (Cambridge, UK) was awarded the British Association of Lung Research prize for work demonstrating that complement protein C5A can impair neutrophil phagocytosis of Staphyloccus aureus in a PI3-kinase dependent manner, ${ }^{3}$ raising speculation that that these mechanisms could be manipulated to alter susceptibility to nosocomial infections. The runner up was Dr Bonvini (London, UK) for her work focusing on the role oestrogen plays in airway sensory nerve activation. $\beta$-oestradiol induced depolarisation of isolated guinea pig vagal nerves ex vivo, but oestrogen receptor antagonist administration had no effect on transient receptor potential ion channel (TRPM)-3-mediated depolarisation, suggesting that the effect of oestrogen on sensory nerve activation may occur upstream of TRPM-3.

The British Lung Foundation award was won by Dr Dickens (Cambridge, UK), who presented her work on cell trafficking defects related to pathogenic surfactant protein $\mathrm{C}$ mutant $173 \mathrm{~T}$ associated with familial pulmonary fibrosis. ${ }^{4}$ These defects may cause type 2 pneumocyte dysfunction and therefore could be important in the development of both familial and sporadic forms of the disease. Finally, Dr Hippolyte (London, UK) received the highly commended BLF award for her work using the UK cystic fibrosis registry data to examine gender differences. Females have worse clinically relevant outcomes including earlier chronic Pseudomonas aeruginosa infection and greater associated lung function decline. ${ }^{5}$

\section{HIGHLIGHTS FROM THORAX}

Four notable studies published in Thorax were showcased at this year's symposium. Dr Jean-Michel Sallenave's group (Paris, France) highlighted that $P$. aeruginosa elastase LasB is a secreted virulence factor that can downregulate the cystic fibrosis transmembrane conductance regulator (CFTR) and degrade immune mediators interleukin (IL)-6 and Trappin-2, important epithelial-derived antimicrobial factors. ${ }^{6}$ Intranasal administration of LasB in mice also induced inflammation, weight loss and death, effects that were rescued by overexpression of $I L-6$ and Trappin-2. These data suggest that targeting LasB could represent a novel therapeutic strategy for patients susceptible to Pseudomonas infections including those with cystic fibrosis and chronic obstructive pulmonary disease (COPD).

Dr Lucile Sese (Bobigny, France) presented thought-provoking data from a prospective cohort study showing that acute exacerbations of idiopathic pulmonary fibrosis (IPF) are significantly temporally associated with an increase in mean level of atmospheric ozone and that exposure to elevated levels of particulate matter $\mathrm{PM}_{10}$ and $\mathrm{PM}_{2.5}$ are associated with increased mortality. ${ }^{\circ}$

Dr Andrey Zinchuk (New Haven, USA) presented a multicentre observational study in which polysomnographic data were used to characterise novel phenotypes of obstructive sleep apnoea (OSA). ${ }^{8}$ Subgroups could be identified that captured risk of adverse cardiovascular outcomes otherwise missed by conventional OSA severity classification criteria. These classification methods could have future importance for risk stratification and treatment selection.

Dr Cecilia O'Kane (Belfast, Northern Ireland) used human in vivo and ex vivo models of lipopolysaccharide-induced inflammation to demonstrate that aspirin could reduce pulmonary neutrophilia and neutrophil-mediated inflammation. ${ }^{9}$ These effects raise speculation that use of aspirin could be beneficial in neutrophil-driven pulmonary 
inflammatory diseases such as acute respiratory distress syndrome (ARDS).

\section{PLENARY SCIENTIFIC}

The plenary scientific session showcases some of the brightest rising stars of UK respiratory academia.

Professor David Kiely (Sheffield, UK) emphasised the importance of a multimodality approach to diagnosis and prognostication in pulmonary hypertension. The potential use of in silico approaches, combined with computational modelling and machine learning, to classify patients could reduce future need for invasive tests.

Dr Charlotte Summers (Cambridge, UK) presented her work on neutrophil biology. Her data, which included some fascinating video images, indicated that neutrophil transit time across the pulmonary circulation is faster than previously thought and that primed neutrophils are retained in the vasculature and subsequently deprime and are rereleased into the systemic circulation. Defects in these processes may underlie development of ARDS.

Dr Robert Snelgrove (London, UK) discussed his work into a novel anti-inflammatory pathway in which leukotriene A4 hydrolase degrades an extracellular matrix-derived neutrophil chemoattractant proline-glycine-proline (PGP). Using a mouse model of allergic airways disease, he demonstrated that PGP accumulation drove pathological epithelial remodelling, mucus production and airway hyper-responsiveness. Subsequently, he demonstrated that PGP is elevated in the sputum of patients with severe asthma, raising speculation that this could provide a viable future therapeutic target.

Finally, Dr John Hurst (London, UK) highlighted the importance of exacerbation risk in COPD, suggesting that an episode of hospitalised exacerbation should be a call to arms for healthcare professionals. He also discussed the importance of disease activity in COPD and raised speculation that small airway imaging could provide an early window to identify active disease that requires intervention.

\section{THE MORRISTON DAVIES LECTURE}

The Morriston Davies lecture was given by Professor John Ioannidis (Stanford, USA), and he proposed a controversial viewpoint that most published clinical research is not useful. The eight criteria of clinically useful research were outlined, which include: problem base, context placement, information gain, pragmatism, patient-centredness, feasibility, transparency and value for money. Importantly, most journals do not consider all eight when assessing submitted manuscripts. Professor Ioannidis additionally highlighted the importance of large-scale collaboration and adoption of replication culture (increased focus on reproducible science).

\section{THE BTS LECTURE}

Professor Sir Michael Marmot (London, UK) gave a rousing lecture on health inequalities and social justice. Describing the links between poverty and exposure to pollution, it becomes easy to understand the striking associations between social deprivation and lung disease across the UK. Professor Sir Marmot described how improving education, housing and household pay could all have lasting impacts on a child's long-term health. In doing so, he created a compelling case for social transfer (government provided social assistance), arguing that, without it, we are simply treating people and then sending them back to the conditions that made them unwell.

\section{PULMONARY VASCULAR DISEASE}

Dr Luke Howard (London, UK) presented the new BTS outpatient pulmonary embolism (PE) guidelines, due to be published this year. Several studies, including randomised controlled trials (RCTs) and meta analyses, have shown that outpatient management of low-risk patients with confirmed PE is as safe and effective as inpatient care. ${ }^{10} 11$ The 11 -point PE severity index (PESI) score is based on easily obtained clinical data and is the most widely validated risk score for PE (although it is not validated for use in pregnancy). The simplified version (s-PESI) is equally able to identify patients at low-risk of mortality. ${ }^{12}$ There is no value in adding brain natriuretic peptide (BNP,) troponin or right ventricular (RV) function assessments to the PESI/sPESI as it does not improve prediction of early mortality, which is key when deciding between outpatient or inpatient care. ${ }^{13}{ }^{14}$ Firstline treatment for confirmed PE is either a direct oral anticoagulant (DOAC) or initial low molecular weight heparin (LMWH) with a subsequent switch to a DOAC, such as dabigatran or edoxaban. There are no data yet for the use of DOACs in cases of suspected PE, but guidelines suggest a DOAC could be used instead of LMWH while awaiting outpatient imaging (ideally within 24 hours).

Dr Gregory Piazza (Boston, USA) discussed different treatment options for acute PE. He highlighted that ultrasound-assisted catheter-directed thrombolysis, which combines low-dose local fibrinolytics and mechanical clot disruptioncan rapidly improves RV function in intermediate ${ }^{15}$ and high-risk patients ${ }^{16}$ with PE. In contrast to the $2 \%$ risk of intracranial haemorrhage associated with systemic thrombolysis, there were no reports of this complication with catheter-directed therapy. ${ }^{17}$ Furthermore, lower dose ( $8 \mathrm{mg}$ tissue plasminogen activator (tPA)) and shorter duration (2 hours) therapy was recently shown to be as effective as standard catheter-directed regimens $(24 \mathrm{mg}$ tPA over 12-24hours) for submassive PE. In some centres in the USA, multidisciplinary PE teams have been founded to evaluate patients and make treatment decisions. ${ }^{18}$ This is especially useful in the care of intermediate/high risk PE, where clinical trial data and guidelines are inadequate. These teams consist of specialists from respiratory medicine, cardiology, vascular surgery, radiology and haematology. It will be interesting to see if there will be a demand for this type of service in the National Health Service (NHS) in the future.

The risk of PE recurrence is significant, even in patients with 'provoked' PE (25\% over 10 years). The EINSTEIN CHOICE study compared $100 \mathrm{mg}$ aspirin to prophylactic and treatment doses of rivaroxaban for prevention of further venous thromboembolism (VTE) in patients with unprovoked and provoked PE. Rivaroxaban reduced the relative risk of VTE by $70 \%(20 \mathrm{mg})$ and $60 \%(10 \mathrm{mg})$ compared with aspirin at 1 year, which raises the question of whether we should be considering long-term anticoagulation in all patients. ${ }^{19}$

\section{INTERSTITIAL LUNG DISEASE}

Patients with sporadic IPF have shorter telomeres compared with age-matched controls, and mutations in telomerase genes have been identified in patients with familial IPF, implicating telomere dysfunction in the pathogenesis of IPF. ${ }^{2021}$ Professor Paul Wolters (San Francisco, USA) showed that deletion of the telomere shelterin protein TRF1 in alveolar epithelial cells (AECs) leads to the spontaneous development of lung fibrosis in mice, suggesting that telomere dysfunction in AECs contributes directly to the development of lung fibrosis. ${ }^{22} \mathrm{He}$ explained that the telomere link may extend beyond IPF and predispose to other fibrotic 
lung diseases. Mutations in telomere genes have been identified in $12 \%$ of patients with rheumatoid arthritis-associated interstital lung disease (ILD), ${ }^{23}$ and telomere length also correlates with survival in chronic hypersensitivity pneumonitis. ${ }^{24}$

Dr Philip Molyneaux (London, UK) argued that infection could be the cause of IPF given that previous studies have shown that therapeutic immunosuppression of patients is harmful. ${ }^{25}$ Until recently, the lungs were thought to be sterile but thanks to new culture-independent techniques we now recognise that the lungs of healthy individuals harbour diverse bacterial populations. Patients with IPF have an increased bacterial burden in bronchoalveolar lavage samples compared with healthy controls and patients with COPD. In patients with IPF, bacterial load at the time of diagnosis correlates with survival. ${ }^{26} 27$ Interestingly, during acute exacerbations, there is a further increase in bacterial burden and significant change in the profile of the microbiome, which is not detected by routine culture. ${ }^{28}$ The results of two ongoing studies examining the effect of antibiotics on IPF outcome (TIPAC 2 and CleanUp IPF) are eagerly awaited.

Professor Martin Kolb (McMaster, Canada) presented the hypothesis that mechanical stretch associated with breathing contributes to disease progression in IPF. ${ }^{29}$ Stretching stiff fibrotic lung tissue leads to the release and activation of the profibrotic cytokine, transforming growth factor (TGF)- $\beta$, which is embedded in the matrix. ${ }^{30}$ This occurs at much lower levels in normal compliant lung. Enhanced TGF- $\beta$ activation contributes to deposition of further matrix proteins, leading to stiffer lungs and a detrimental feed-forward loop. This could explain why fibrosis has a propensity to develop in the subpleural regions where the pressure change and stretch is greatest.

Dr Billy Fahy (Stevenage, UK) explained that, in the future, biomarkers could be used to differentiate subsets of patients with IPF and guide therapeutic decisions. However, there are no biomarkers currently in clinical use. He spoke about the challenges of biomarker identification and how machine learning is being used to identify biomarker signatures in large datasets. Using data from the longitudinal PROFILE (Prospective study of Fibrosis in Lung Endpoints) cohort an epithelial biomarker signature was recently shown to predict disease progression and death in IPF. ${ }^{31}$

While IPF grabbed a lot of the headlines, we also heard from Dr Vijay Maharajan (Cambridge, UK) who described ways to automate the analysis of cystic lung disease. Splitting the lung into zones could allow accurate scoring of cyst distribution and differentiate between diseases. The automated counting and assessment could also play a role in long-term follow-up and clinical trial design.

\section{ASTHMA}

Professor Maria Belvisi (London, UK) focused on the non-allergic influences on asthma. Many non-allergic asthma triggers, such as pollution and tobacco, can cause cough and breathlessness, symptoms that are largely unresponsive to current treatments targeting inflammation. She hypothesised that irritants directly activate transient receptor potential (TRP) ion channels to initiate these sensory reflex events. She highlighted that TRPV4 activation releases ATP which, in turn, acts on $\mathrm{P} 2 \times 3$ present on sensory nerves, resulting in the cough reflex. ${ }^{32}$ The discovery of this axis opens up a completely new therapeutic opportunity for improving symptoms with use of either P $2 \times 3$ or TRPV4 antagonists. $^{33}$

Professor Adnan Custovic (London, UK) showed that the effect of early-life exposures (eg, to cat) on sensitisation and asthma differs over time, rendering the generalisation of effects from cross-sectional analyses misleading. ${ }^{34}$ Therefore, the findings of apparently contradictory cross-sectional studies may be due to markedly different trajectories of clinical outcomes between individuals exposed or not exposed in early life. ${ }^{34} \mathrm{He}$ showed that mite allergen avoidance in children, using allergen-proof bedding, reduces emergency hospital attendance with severe asthma exacerbations ${ }^{35}$ and that temperature-controlled laminar airflow devices in real-life studies reduce asthma exacerbation rates. ${ }^{36}$ This raises the question of whether asthma guidelines should reassess the role of allergen avoidance as a treatment option. More controversially, he challenged the dogma that allergen desensitisation is ineffective in asthma control. Sublingual immunotherapy (SLIT) is certainly safe, and there is an increasing body of evidence that dust mite SLIT tablet may be clinically effective in adults with partially controlled allergic asthma. ${ }^{37}$ Grass SLIT tablet may also modify the natural history of airway symptoms in grass-allergic children ${ }^{38}$

Professor Liam Heaney (Belfast, Northern Ireland) described the progress on the UK MRC (Medical Research Council) RASP (refracrtory asthma stratification project) programme on severe asthma. ${ }^{39}$ The aim of this programme is to understand disease control using composite endpoints and by assessing drug adherence using $\mathrm{FeNO}$ and the inhaler compliance assessment device. This could enable the down-titration of medication where feasible. He described the wide range of novel biologicals competing for a narrow market although with potentially major health economic benefits through exacerbation reduction.

Dr Stephen Fowler (Manchester, UK) contrasted and compared the BTS/SIGN (Scottish Intercollegiate guidelines network) guidelines with new National Institute for Health and Care Excellence (NICE) asthma guidelines. There is some agreement, but the NICE guideline uses FeNO as part of an unvalidated complex web-based diagnostic pathway and does not take into account the age and gender differences in the FeNO normal range. In addition, NICE guidance does not include important topics such as smoking, comorbidities, devices or pregnancy. There was agreement that both guidelines were based on efficacy RCTs in unrepresentative populations. We therefore urgently need clinical effectiveness data in broader populations on which to base future guidelines.

\section{CHRONIC OBSTRUCTIVE PULMONARY DISEASE}

Mechanistic aspects of COPD were focused on at this year's meeting with intriguing data from Lodge $e t a l^{40}$ (Cambridge, UK) showing that hypoxia increases neutrophil elastase in a PI3-kinase-dependent manner, effects that are further augmented during exacerbations. Evaluation of the neutrophil secretome in hypoxic conditions showed several upregulated proteins that may contribute to tissue damage. These proteins could represent future therapeutic targets. Ryan et al (Edinburgh, UK) highlighted mechanisms of impaired macrophage efferocytosis in COPD. Sulforaphane, an agonist of the transcription factor Nrf2 (which regulates antioxidant response elements), was shown to partially rescue defective efferocytosis in monocyte-derived and alveolar macrophages from patients with COPD. ${ }^{41}$

Gillpin et al (Belfast, Northern Ireland) presented work looking at the effects of cigarette smoke and electronic cigarette vapour extracts on growth and biofilm formation of COPD-relevant bacteria including Haemophilus influenzae and Pseudomonas aeruginosa in vitro. They demonstrated that both cigarette smoke and electronic cigarette vapour extract enhanced inflammatory responses to bacteria and increased biofilm formation. ${ }^{42}$ 
It remains to be seen whether these potentially adverse effects of electronic cigarette vapour observed in vitro also occur in vivo.

\section{INFECTION}

Dr Abubakar et al (London, UK) presented data from PredictTB a large UK multicentre study. ${ }^{43}$ In this study, subjects who were new migrants or contacts of active TB cases were screened for latent TB. There were 97 out of 9610 participants (1\%) who developed active TB during the study. The authors identified that using either interferon-gamma release assay- based tests or tuberculin skin test with a $15 \mathrm{~mm}$ threshold were best suited to screening in low-risk populations.

Dr Szylar et al (London, UK) evaluated the effects of regulatory $\mathrm{T}$ cells (Tregs) on macrophage inflammatory responses to Streptococcus pneumoniae. ${ }^{44}$ Coculture of monocyte-derived macrophages (MDM) with Tregs reduced MDM production of tumour necrosis factor- $\alpha$ and IL- 6 . They also demonstrated, using a novel human in vivo model of pneumococcal challenge involving intradermal injection of UV-killed S. pneumoniae, that Tregs accumulate at the site of injection. These preliminary findings suggest Tregs could be important in modulating macrophage inflammatory responses to $S$. pneumoniae.

Dr Plowright et al (Nottingham, UK) evaluated outcomes following cardiovascular events in a large cohort of 101597 patients with bronchiectasis. ${ }^{45}$ After adjusting for age and sex, there was no difference in in-hospital mortality following acute myocardial infarction, coronary artery bypass graft or percutaneous angioplasty in individuals with bronchiectasis compared with the general population. The presence of bronchiectasis was, however, associated with longer mean hospital stay.

\section{SMOKING}

Dr Sanjay Agrawal (Leicester, UK) argued that there is no strong evidence to suggest that that cigarette smokers who use use e-cigarettes are less likely to quit smoking. Recent data suggest that vaping does increase the success of quit rates. ${ }^{46} \mathrm{E}$-cigarettes are generally thought to be a safer alternative to smoking traditional cigarettes, but little is known about the long-term effects of vaping on lung health.

There are $>7000$ different flavours of e-liquids available in the USA, all with very different chemical compositions. There is no regulation or inhalational toxicity testing performed prior to their release onto the market, which is a cause for concern. Dr Robert Tarran (North Carolina, USA) showed data demonstrating the toxic effects of different flavour e-liquids on human airway epithelial cells in vitro. ${ }^{47}$ Examination of sputum from healthy smokers and vapers demonstrates raised levels of proteases and mucins compared with controls, which raises the possibility that vaping could also cause emphysema. ${ }^{48} \mathrm{He}$ also showed bronchoscopic images indicating marked erythema in the airways of e-cigarette users. He suggested this may be due to direct heat damage from e-cigarettes, but further studies are required to investigate this.

Dr Rachel Murray (Nottingham, UK) described the challenges to successfully implementing smoking cessation in the NHS. In 2015/2016 475000 hospital admissions were directly smoking-related. Moreover, even greater numbers of current smokers are admitted to hospital every year (1.1 million smokers in 2010/2011). This represents a huge number of opportunities to intervene and promote smoking cessation. Smoking cessation interventions are the most effective method available, but stop smoking services have been badly hit by funding cuts. ${ }^{49} \mathrm{Dr}$ Murray argued that, although NICE guidance and Department of Health policy outline key interventions for smoking cessation without regulatory drivers, there is no incentive for Trusts to deliver.

Contributors All authors contributed to writing of the manuscript.

Funding The authors have not declared a specific grant for this research from any funding agency in the public, commercial or not-for-profit sectors.

Competing interests None declared.

Patient consent Not required.

Provenance and peer review Commissioned; externally peer reviewed.

(c) Article author(s) (or their employer(s) unless otherwise stated in the text of the article) 2018. All rights reserved. No commercial use is permitted unless otherwise expressly granted.

\section{REFERENCES}

1 Wortley MA DE, Bonvin MA. Bacteria can trigger airway sensory nervias via the activation of TLR2. Thorax 2017;72(Suppl 3):A1.

2 Walsted ES, Faisal A, Jolley CJ, et al. Increased respiratory neural drive and work of breathing in exercise-induced laryngeal obstruction. J App/ Physiol 2018;124:356-63.

3 Wood AV AJT, Okkenhaug K, Scott J, et al. T5 Complement protein c5a induces prolonged neutrophil dysfunction in a clinically relevant model of human bacteraemia. Thorax 2017;72(Suppl 3):A3.

4 Dickens ME JA, Marciniak SJ. T6 Understanding the cellular dysfunction caused by pathogenic surfactant protein c mutant i73t. Thorax 2017;72(Suppl 3):A4-5.

5 Hippolyte NS SS, Bilton D, Griesenbach U, et al. Diabetes and pseudomonas, a terrible combination? Examining the uk cystic fibrosis registry for a sex difference in outcomes (2008-2013). Thorax 2017;72(Suppl 3):A2-3.

6 Saint-Criq V, Villeret B, Bastaert F, et al. Pseudomonas aeruginosa LasB protease impairs innate immunity in mice and humans by targeting a lung epithelial cystic fibrosis transmembrane regulator-IL-6-antimicrobial-repair pathway. Thorax 2018;73:49-61.

7 Sesé L, Nunes H, Cottin V, et al. Role of atmospheric pollution on the natural history of idiopathic pulmonary fibrosis. Thorax 2018;73:145-50.

8 Zinchuk AV, Jeon S, Koo BB, et al. Polysomnographic phenotypes and their cardiovascular implications in obstructive sleep apnoea. Thorax 2018;73.

9 Hamid U, Krasnodembskaya A, Fitzgerald M, et al. Aspirin reduces lipopolysaccharideinduced pulmonary inflammation in human models of ARDS. Thorax 2017;72:971-80.

10 Aujesky D, Roy PM, Verschuren F, et al. Outpatient versus inpatient treatment for patients with acute pulmonary embolism: an international, open-label, randomised, non-inferiority trial. Lancet 2011;378:41-8.

11 Zondag W, Kooiman J, Klok FA, et al. Outpatient versus inpatient treatment in patients with pulmonary embolism: a meta-analysis. Eur Respir J 2013;42:134-44.

12 Righini M, Roy PM, Meyer G, et al. The Simplified Pulmonary Embolism Severity Index (PESI): validation of a clinical prognostic model for pulmonary embolism. J Thromb Haemost 2011;9:2115-7.

13 Lankeit M, Jiménez D, Kostrubiec M, et al. Predictive value of the high-sensitivity troponin T assay and the simplified Pulmonary Embolism Severity Index in hemodynamically stable patients with acute pulmonary embolism: a prospective validation study. Circulation 2011;124:2716-24.

14 den Exter PL, Zondag W, Klok FA, et al. Efficacy and safety of outpatient treatment based on the hestia clinical decision rule with or without $\mathrm{N}$-terminal pro-brain natriuretic peptide testing in patients with acute pulmonary embolism. a randomized clinical trial. Am J Respir Crit Care Med 2016;194:998-1006.

15 Kucher N, Boekstegers P, Müller OJ, et al. Randomized, controlled trial of ultrasoundassisted catheter-directed thrombolysis for acute intermediate-risk pulmonary embolism. Circulation 2014;129:479-86.

16 Piazza G, Hohlfelder B, Jaff MR, et al. A prospective, single-arm, multicenter trial of ultrasound-facilitated, catheter-directed, low-dose fibrinolysis for acute massive and submassive pulmonary embolism: the SEATTLE II study. JACC Cardiovasc Interv 2015;8:1382-92

17 Meyer G, Vicaut E, Danays T, et al. Fibrinolysis for patients with intermediate-risk pulmonary embolism. N Engl J Med 2014;370:1402-11.

18 Dudzinski DM, Piazza G. Multidisciplinary pulmonary embolism response teams. Circulation 2016;133:98-103.

19 Weitz Jl, Lensing AWA, Prins MH, et al. Rivaroxaban or aspirin for extended treatment of venous thromboembolism. N Engl J Med 2017;376:1211-22.

20 Alder JK, Chen JJ, Lancaster L, et al. Short telomeres are a risk factor for idiopathic pulmonary fibrosis. Proc Natl Acad Sci U SA 2008;105:13051-6.

21 Armanios MY, Chen JJ, Cogan JD, et al. Telomerase mutations in families with idiopathic pulmonary fibrosis. N Engl J Med 2007;356:1317-26.

22 Naikawadi RP, Disayabutr S, Mallavia B, et al. Telomere dysfunction in alveolar epithelial cells causes lung remodeling and fibrosis. JCI Insight 2016;1:e86704 
23 Juge PA, Borie R, Kannengiesser C, et al. Shared genetic predisposition in rheumatoid arthritis-interstitial lung disease and familial pulmonary fibrosis. Eur Respir J 2017;49:1602314.

24 Ley B, Newton CA, Arnould I, et al. The MUC5B promoter polymorphism and telomere length in patients with chronic hypersensitivity pneumonitis: an observational cohortcontrol study. Lancet Respir Med 2017;5:639-47.

25 Raghu G, Anstrom KJ, King TE, et al. Prednisone, azathioprine, and N-acetylcysteine for pulmonary fibrosis. N Eng/ J Med 2012;366:1968-77.

26 Molyneaux PL, Cox MJ, Willis-Owen SA, et al. The role of bacteria in the pathogenesis and progression of idiopathic pulmonary fibrosis. Am J Respir Crit Care Med 2014;190:906-13.

27 Molyneaux PL, Willis-Owen SAG, Cox MJ, et al. Host-microbial interactions in idiopathic pulmonary fibrosis. Am J Respir Crit Care Med 2017;195:1640-50.

28 Molyneaux PL, Cox MJ, Wells AU, et al. Changes in the respiratory microbiome during acute exacerbations of idiopathic pulmonary fibrosis. Respir Res 2017;18:29.

29 Hinz B, Suki B. Does breathing amplify fibrosis? Am J Respir Crit Care Med 2016;194:9-11.

30 Froese AR, Shimbori C, Bellaye PS, et al. Stretch-induced activation of transforming growth factor- $\beta 1$ in pulmonary fibrosis. Am J Respir Crit Care Med 2016;194:84-96.

31 Maher TM, Oballa E, Simpson JK, et al. An epithelial biomarker signature for idiopathic pulmonary fibrosis: an analysis from the multicentre PROFILE cohort study. Lancet Respir Med 2017;5:946-55.

32 Bonvini SJ, Birrell MA, Grace MS, et al. Transient receptor potential cation channel, subfamily $\mathrm{V}$, member 4 and airway sensory afferent activation: Role of adenosine triphosphate. J Allergy Clin Immunol 2016;138:249-61.

33 Belvisi MG, Birrell MA. The emerging role of transient receptor potential channels in chronic lung disease. Eur Respir J 2017;50:1601357.

34 Ihuoma H, Belgrave DC, Murray CS, et al. Cat ownership, cat allergen exposure, and trajectories of sensitization and asthma throughout childhood. J Allergy Clin Immunol 2018;141.

35 Murray CS, Foden P, Sumner H, et al. Preventing severe asthma exacerbations in children. A randomized trial of mite-impermeable bedcovers. Am J Respir Crit Care Med 2017:196:150-8

36 Schauer U, Bergmann KC, Gerstlauer M, et al. Improved asthma control in patients with severe, persistent allergic asthma after 12 months of nightly temperaturecontrolled laminar airflow: an observational study with retrospective comparisons. Eur Clin Respir J 2015:2:28531.
37 Virchow JC, Backer V, Kuna P, et al. Efficacy of a house dust mite sublingual allergen immunotherapy tablet in adults with allergic asthma: a randomized clinical trial. JAMA 2016;315:1715-25.

38 Valovirta E, Petersen TH, Piotrowska T, et al. Results from the 5-year SQ grass sublingual immunotherapy tablet asthma prevention (GAP) trial in children with grass pollen allergy. J Allergy Clin Immunol 2018;141.

39 Heaney LG, Djukanovic R, Woodcock A, et al. Research in progress: Medical Research Council United Kingdom Refractory Asthma Stratification Programme (RASP-UK). Thorax 2016;71:187-9.

40 Lodge KH KM, Robbins AJ, Chilvers ER, et al. S114 Hypoxia drives neutrophilmediated endothelial damage in copd. Thorax 2017;72(Suppl 3):A69-70.

41 Ryan RB EM, Bewley, P Coelho MA, Rumsey W, et al. S115 Mechanisms to reverse impaired macrophage efferocytosis in copd. Thorax 2017;72(Suppl 3):A70.

42 Gilpin KM DF, Gallagher K, Bengoechea J, et al. The effect of cigarette and electronic cigarette vapour on bacteria in chronic lung infection. Thorax 2017;72(Suppl 3):A71.

43 Abubakar FD I, Southern J, Sitch AJ, et al. S31 Prognostic value of interferon gamma release assays and tuberculin skin test in predicting the development of active tuberculosis: the uk predict tb cohort study. Thorax 2017;72(Suppl 3):A22.

44 Szylar JB G. S10 Suppression of macrophage inflammatory responses to streptococcus pneumoniae by regulatory t cells. Thorax 2017;72(Suppl 3):A9-10.

45 Plowright JH M, Quint JK, Hubbard RB, et al. S43 In-hospital mortality and length of stay following cardiovascular events and interventions in people with bronchiectasis: a population based study. Thorax 2017;72(Suppl 3):A29.

46 Beard E, West R, Michie S, et al. Association between electronic cigarette use and changes in quit attempts, success of quit attempts, use of smoking cessation pharmacotherapy, and use of stop smoking services in England: time series analysis of population trends. BMJ 2016:354:i4645.

47 Rowell TR, Reeber SL, Lee SL, et al. Flavored e-cigarette liquids reduce proliferation and viability in the CALU3 airway epithelial cell line. Am J Physiol Lung Cell Mol Physiol 2017;313:L52-L66.

48 Reidel B, Radicioni G, Clapp PW, et al. E-cigarette use causes a unique innate immune response in the lung, involving increased neutrophilic activation and altered mucin secretion. Am J Respir Crit Care Med 2018;197:492-501.

49 Murray RL, Leonardi-Bee J, Marsh J, et al. Systematic identification and treatment of smokers by hospital based cessation practitioners in a secondary care setting: cluster randomised controlled trial. BMJ 2013;347:f4004. 